Research Article

\title{
Study on the Expression Differences and the Correlation with H2BE Gene of Th Related Cytokines in SSDHS and LDSDS TCM-Syndromes of CHB Patients
}

\author{
Chao Liu $(\mathbb{D}$, Yanfeng Zheng, Xia Li, Baixue Li, Li Wen, Dong Wang, Quansheng Feng $\mathbb{D}$, \\ and Cen Jiang $\mathbb{D}$
}

College of Basic Medical Sciences, Chengdu University of Traditional Chinese Medicine, Chengdu 610075, China

Correspondence should be addressed to Quansheng Feng; fengqscdutcm@126.com and Cen Jiang; jiangcencdutcm@126.com

Received 24 July 2020; Revised 9 October 2020; Accepted 28 January 2021; Published 22 February 2021

Academic Editor: Weicheng $\mathrm{Hu}$

Copyright (c) 2021 Chao Liu et al. This is an open access article distributed under the Creative Commons Attribution License, which permits unrestricted use, distribution, and reproduction in any medium, provided the original work is properly cited.

\begin{abstract}
Although our previous studies revealed that H2BE exhibited significantly differential expression between two CHB TCMsyndromes: Spleen-stomach dampness-heat syndrome, SSDHS and liver-depression spleen-deficiency syndrome, LDSDS, the underlying mechanisms remain largely unknown. Recent studies showed that dynamic expression fluctuation of Th related cytokines in CHB TCM-syndromes, and furthermore, their expression levels were largely regulated by H2BE. This study aims to detect the expression level differences of Th related cytokines between these two TCM-syndromes and further investigate the underlying regulatory mechanisms. The expression levels of the four Th related cytokines and H2BE were analyzed and the protein-protein interaction networks between $\mathrm{H} 2 \mathrm{BE}$ and the four cytokines were constructed. Our results suggested that almost all the cytokines were significantly upregulated compared with the healthy group $(P<0.05)$. Interestingly, among the four cytokines, only IL-4 and INF- $\gamma$ showed statistical significance between these two syndromes. The protein-protein interaction networks demonstrated that H2BE was indirectly associated with IL-4 and IL-10, and H2BE may regulate the expression levels of cytokines through GATA3. Taken together, our results indicated that IL- 4 and INF- $\gamma$ are two representative cytokines that may serve as two potential biochemical indicators of SSDHS and LDSDS in CHB patients; except what has been reported, our study found that one possible way for H2BE to regulate the expression of cytokines is to interact with GATA3 directly or indirectly.
\end{abstract}

\section{Introduction}

Chronic hepatitis $\mathrm{B}(\mathrm{CHB})$ is a serious worldwide health problem that is often tightly associated with serious liver diseases, such as liver cirrhosis and even hepatocellular carcinoma (HCC) [1]. It was reported that nearly $20-30 \%$ of chronic hepatitis patients could further lead to cirrhosis or HCC due to the failure of virus clearance, and $15-40 \%$ of chronic patients will progress to cirrhosis and even HCC without any treatments [2, 3]. Globally, around 257 million people are suffering HBV, and sexual, blood and perinatal are the three major modes of HBV transmission $[4,5]$. It is worth noting that among the three transmission modes, vertically from mother to child is the major cause for chronic HBV infection which account for $80-90 \%$ of cases of chronic
HBV infection, and nearly $5-10 \%$ cases were infected during adolescence and adulthood $[1,6]$. Therefore, CHB has become a serious disease affecting human health.

For CHB treatment, the goal is to improve the life quality and prevent the formation of cirrhosis and HCC, and unfortunately, there is still no specific medicine that could cure it completely in western medicine. Chinese Traditional Medicine (TCM), as complementary and alternative medicine, is becoming more and more popular in treating complex diseases [7]. In addition, TCM treatments for some chronic diseases, such as cancer and $\mathrm{CHB}$, especially when combined with western medicine, could produce incredible effects $[8,9]$. TCM-syndrome, which is also called "ZHENG" in Mandarin Chinese, is the basic unit and the main perspective in TCM, which could help us understand the body 
homeostasis and provide guidance for the treatment of diseases $[10,11]$. In some Asian countries, TCM based on syndrome has been used to diagnose and guide the treatment of CHB for many years [12]. Patients who suffer from an identical disease would be treated with different methods based on the TCM-syndromes and vice versa. Different TCM-syndromes that received the same therapy could exhibit various responses [13]. Furthermore, utilizing TCM without taking syndrome classification into account could generate serious side effects [14]. On the other hand, until now, TCM-syndromes justification still mainly relies on experienced practitioners, which may lead to ambiguity and subjectivity for one syndrome by different doctors [12]. However, in reality, modern medicine has been closely combined with TCM, such as the biochemical indicators from modern clinical examination have been widely used in TCM diagnosis, and that is why patients often receive a biochemical indicator inspection report in a TCM clinic. These biochemical indicators could reflect the physical state more objectively than TCM-syndrome diagnosed by experienced practitioners. Therefore, establishing the relationship between these biochemical indicators with TCMsyndromes will make great significance for the development of TCM. Fortunately, considerable achievements have been made in this field [15].

Spleen-stomach dampness-heat syndrome (SSDHS) and liver-depression spleen-deficiency syndrome (LDSDS) are two common TCM-syndromes for CHB. A large sample investigation conducted by our team found that among 1260 CHB patients, $16.7 \%$ were SSDHS and $8.8 \%$ were LDSDS [16]. Thus, looking for effective biochemical indicators to differentiate SSDHS and LDSDS could be quite helpful for clinical considerations. In addition, the previous mRNA screening for the CHB two TCM-syndromes (SSDHS and LDSDS) in our laboratory found that there were 9 differentially expressed genes between the two syndromes. Among them, the expression of HIST2H2BE (H2BE) was the most significant one [17]. Studies found that the expression levels of histone could participate in many cellular processes, such as DNA replication, repair, recombination, transcriptional regulation, etc. [18, 19]. Further studies confirmed that histone fragments encoded by H2BE could act on the immune dominant epitopes in Th cells and then further stimulate the proliferation, differentiation, and IL-2 release of Th cells [20]. Under the stimulation of hepatitis B antigen, Th cells then mainly differentiate into two subgroups, Th1 and Th2. Th1 mainly participates in the cellular immune response by secreting interleukin-2 (IL-2) and INF$\gamma$, and Th2 is mainly involved in the humoral immune response by secreting interleukin-4 (IL-4), interleukin-10 (IL-10) [21]. Thus, the equilibrium state of Th1/Th2 is considered as an important effective factor of hepatitis B chronicity [22].

Collectively, we suggested that the differential expression of H2BE in SSDHS and LDSDS could further lead to the ratio of Th1/Th2 change and then making SSDHS and LDSDS possess the different levels of cytokines. In order to investigate whether there is any relationship between $\mathrm{CHB}$ TCM-syndromes and cytokines, in this paper, we chose IL-2,
IL-4, IL-10, and INF- $\gamma$ as the research objects and examined the expression level of H2BE, IL-2, IL-4, IL-10, and INF- $\gamma$. Furthermore, the correlation between TCM-syndromes and the specific cytokines was analyzed. Our results could provide more details for the causes of the same disease with different TCM-syndromes and a reference for the further development of biochemical indicators of SSDHS and LDSDS syndromes.

\section{Materials and Methods}

2.1. Participants. A total of $31 \mathrm{CHB}$ patients were enrolled from August 2018 to February 2019 in the Outpatient Department of Hepatology, General Hospital of Chengdu Military Region. Among the $31 \mathrm{CHB}$ patients, there were 11 SSDHS CHB patients, 11 LDSDS, and 9 healthy volunteers (control group, CG). All the enrolled $\mathrm{CHB}$ patients underwent rigorous screening according to the selection criteria. The inclusion criteria were as follows:

(1) CHB patients' TCM-syndrome was differentiated by Chinese physicians with the title of associate professor or above

(2) The age of these cases who met the selection criteria in this study was between 18 and 65, male or female

(3) In addition, according to the diagnostic criteria in the 2015 guidelines for the prevention and treatment of chronic hepatitis B in China, CHB patients in this study were HBsAg or HBV-DNA positive for at least six months

The details of the samples are listed in Table 1. The TCMsyndrome types were identified according to the TCMsyndrome standards of chronic hepatitis B established by the Chinese Society of Traditional Chinese Medicine, 2017 [23]. This study was approved by the Medical Ethics Committee of Affiliated Hospital of Chengdu University of Traditional Chinese Medicine, China.

2.2. RNA Isolation and $q R T-P C R$ Analysis. All the blood samples were collected from the participants' peripheral venous blood, and the samples were frozen in liquid nitrogen immediately and then stored at $-80^{\circ} \mathrm{C}$. Total RNAs were extracted by using TRIzol ${ }^{\circledR}$ Reagent (Life Technologies, USA) according to the manufacturer's protocol. The primers used for qRT-PCR analyses are listed in Table 2. CDNAs were synthesized by using the SuperScript ${ }^{\mathrm{TM}}$ III First-Strand Synthesis System (Life Technologies, USA). SYBR-based qRT-PCR $\left(\mathrm{SYBR}^{\circledR}\right.$ Green Master Mix, Life Technologies, USA) were performed on a step-one real-time system (ABI company) by using the following reaction conditions: predenaturation at $95^{\circ} \mathrm{C}$ for $2 \mathrm{~min}$, followed by 40 cycles of $95^{\circ} \mathrm{C}$ for $10 \mathrm{~s}$ and $60^{\circ} \mathrm{C}$ for $10 \mathrm{~s}, 40$ cycles. Each sample was performed in three technical triplicates.

2.3. Serum Cytokine Measurements. Serum samples from 11 SSDHS, 11 LDSDS, and 9 healthy volunteers were collected by centrifuging at $3500 \mathrm{r} / \mathrm{min}, 4^{\circ} \mathrm{C}$ for $10 \mathrm{~min}$, and stored at 
TABLE 1: Baseline comparison of the clinical characteristics among the groups.

\begin{tabular}{lcccc}
\hline Group & Number of cases & Male & Gender $^{\mathrm{a}}$ & Female $^{*}$ \\
\hline LDSDS & & $5(45.5 \%)$ & $6(54.5 \%)$ & $39.27 \pm 8.661^{\mathrm{c}}$ \\
SSDHS & 11 & $7(63.6 \%)$ & $4(36.4 \%)$ & $36.00 \pm 12.578^{\mathrm{c}}$ \\
CG $^{\mathrm{d}}$ & 9 & $4(44.4 \%)$ & $5(55.6 \%)$ & $33.56 \pm 8.00^{\mathrm{c}}$ \\
Total & 31 & $16(57.69 \%)$ & $15(42.31 \%)$ & - \\
\hline
\end{tabular}

${ }^{\mathrm{a}}$ There was no significant difference in gender composition among the three groups by the chi-square test $(P>0.05)$. ${ }^{\mathrm{b}}$ There was no significant difference in age composition among the three groups by one-way analysis of variance $(P>0.05)$. ${ }^{c}$ mean \pm standard deviation. ${ }^{\mathrm{d}}$ Healthy group (control group).

TABLE 2: The primer pairs for qRT-PCR validation.

\begin{tabular}{lc}
\hline Primers & Sequence $\left(5^{\prime}-3^{\prime}\right)$ \\
\hline H2BE-F & agtggctgagttcggctgtc \\
H2BE-R & gctgccaagcgtcagtcata \\
U6b-F & ggcagtcgaccgacgaata \\
U6b-R & cgtgaaagaccgcagcaa \\
\hline
\end{tabular}

$-80^{\circ} \mathrm{C}$. Four Th related cytokines (IL-2, IL-4, IL-10, INF- $\gamma$ ) were detected using human cytokines ELISA kits (Life Technologies, USA) based on the manufacturer's protocol. The optical density values of the four cytokines were determined by using a Labsystems Multiskan MS Reader. The concentration of each cytokine was calculated using a standard curve.

\subsection{The Protein-Protein Interaction Networks Construction.} The protein-protein interaction networks between $\mathrm{H} 2 \mathrm{BE}$ and the four cytokines were constructed by using the GeneMANIA database (http://genemania.org/). Briefly, input the gene name of HIST2H2BE, IL-2, IL-4, and IL-10, respectively, and then click the search button to construct the networks.

2.5. Statistical Analysis. Data of qRT-PCR were processed using Microsoft 2010 based on the $2^{-\Delta \Delta \mathrm{Ct}}$ method and the CG group was set as the control. The significance between the experimental groups (LDSDS, SSDHS) and control group (CG) was detected by using the one-way ANOVA method via the SPSS 24.0. In addition, the expression levels of the four cytokines were also analyzed using SPSS 24.0. The standards for the potential marker selection are that the potential marker which could not only distinguish the two syndromes but also could distinguish the syndromes from the healthy group (the significance condition is at least $P$ value $<0.05)$.

\section{Results}

3.1. Patient Characteristics. A total of 31 participants were enrolled in this study, including 11 LDSDS, 11 SSDHS, and 9 healthy subjects. Among the 11 LDSDS, 5 were males and 6 were females; among the 11 SSDHS, 7 were males and 4 were females; among the 9 healthy participants, there were 4 males and 5 females (Table 1). There was no significant difference in age at baseline (Table 1).
3.2. Cytokines Expression Level Analysis. To ascertain the expression levels of cytokines between $\mathrm{CHB}$ patients and healthy subjects, and also to identify the differently expressed cytokines between the LDSDS and SSDHS, the expression levels of the four cytokines were detected by ELISA. The results showed that the expression levels of IL-2 were significantly increased $(P<0.01)$ in LDSDS and SSDHS compared with the healthy group; however, there is no significant difference observed $(P>0.05)$ between LDSDS and SSDHS (Table 3). For IL-4, both LDSDS and SSDHS exhibited increased levels $(P<0.05)$ compared with the healthy group (Table 3 ). Significant increase of IL-10 was observed only in LDSDS, but not in SSDHS $(P<0.01)$ when compared with the healthy group, and there is a statistical difference $(P<0.05)$ between LDSDS and SSDHS. Finally, the expression levels of INF- $\gamma$ in different groups were analyzed. Results showed that the expression levels of INF- $\gamma$ in LDSDS and SSDHS showed a dramatic increase $(P<0.05)$ compared with the healthy group. Notably, the expression level of INF- $\gamma$ in LDSDS was even increased compared with SSDHS $(P<0.01)$ (Table 3$)$. Collectively, what attracts us most is that among the four cytokines, only IL- 4 and INF- $\gamma$ showed statistical differences not only between these two syndromes but also between the syndromes and healthy group, suggesting that these two cytokines may serve as the potential biochemical indicators of the TCM-syndromes of $\mathrm{CHB}$, but these need to be further studied.

3.3. Analysis of H2BE Gene Expression Level. In order to illuminate the expression levels of H2BE in LDSDS, SSDHS, and the healthy group, we performed the qRT-PCR. QRT-PCR results showed that LDSDS-10 exhibited the highest expression level in the LDSDS group, while the lowest expression level was LDSDS-6. In the SSDHS group, SSDHS-10 showed the highest expression level and SSDHS-3 exhibited the lowest. Interestingly, the expression levels of H2BE in LDSDS were generally higher than that in SSDHS, which was consistent with the cytokines results (Figure 1). Moreover, when compared with the CG group, the expression levels of the $\mathrm{H} 2 \mathrm{BE}$ in most of the $\mathrm{CHB}$ patients showed a trend of increase, suggesting that the expression level of $\mathrm{H} 2 \mathrm{BE}$ may be related to the formation of TCM-syndromes to some extent, but this needs to be further studied in the following research.

3.4. Correlation Analysis between $H 2 B E$ and the Cytokines. In order to further elucidate how H2BE works on the cytokines and also mine the latent genes which participate in 
TABLE 3: Expression levels of the four cytokines detected with ELISA.

\begin{tabular}{|c|c|c|c|}
\hline Cytokines & LDSDS & SSDHS & CG \\
\hline IL-2 & $133.451 \pm 20.078^{※ ※}$ & $121.870 \pm 21.934 ※ ※$ & $76.308 \pm 14.061^{\mathrm{a}}$ \\
\hline IL-4 & $68.194 \pm 10.387^{※ ※ \# \# ~}$ & $50.432 \pm 7.936^{※}$ & $39.618 \pm 7.953^{\mathrm{a}}$ \\
\hline IL-10 & $123.728 \pm 26.204^{※ ※ \#}$ & $93.452 \pm 13.689$ & $81.416 \pm 10.387^{\mathrm{a}}$ \\
\hline INF- $\gamma$ & $159.037 \pm 33.737$ ※\#\# & $106.233 \pm 23.602^{※}$ & $80.097 \pm 16.610^{\mathrm{a}}$ \\
\hline
\end{tabular}

a mean \pm standard deviation. ${ }^{*}$ The difference was statistically significant compared with the healthy group $(P$ value $<0.05)$. ${ }^{*}$ The difference was statistically significant compared with the healthy group $(P$ value $<0.01)$. ${ }^{\#}$ The difference was statistically significant compared with the SSDHS group $(P$ value $<0.05) .{ }^{\# \#}$ The difference was statistically significant compared with the SSDHS group $(P$ value $<0.01)$.

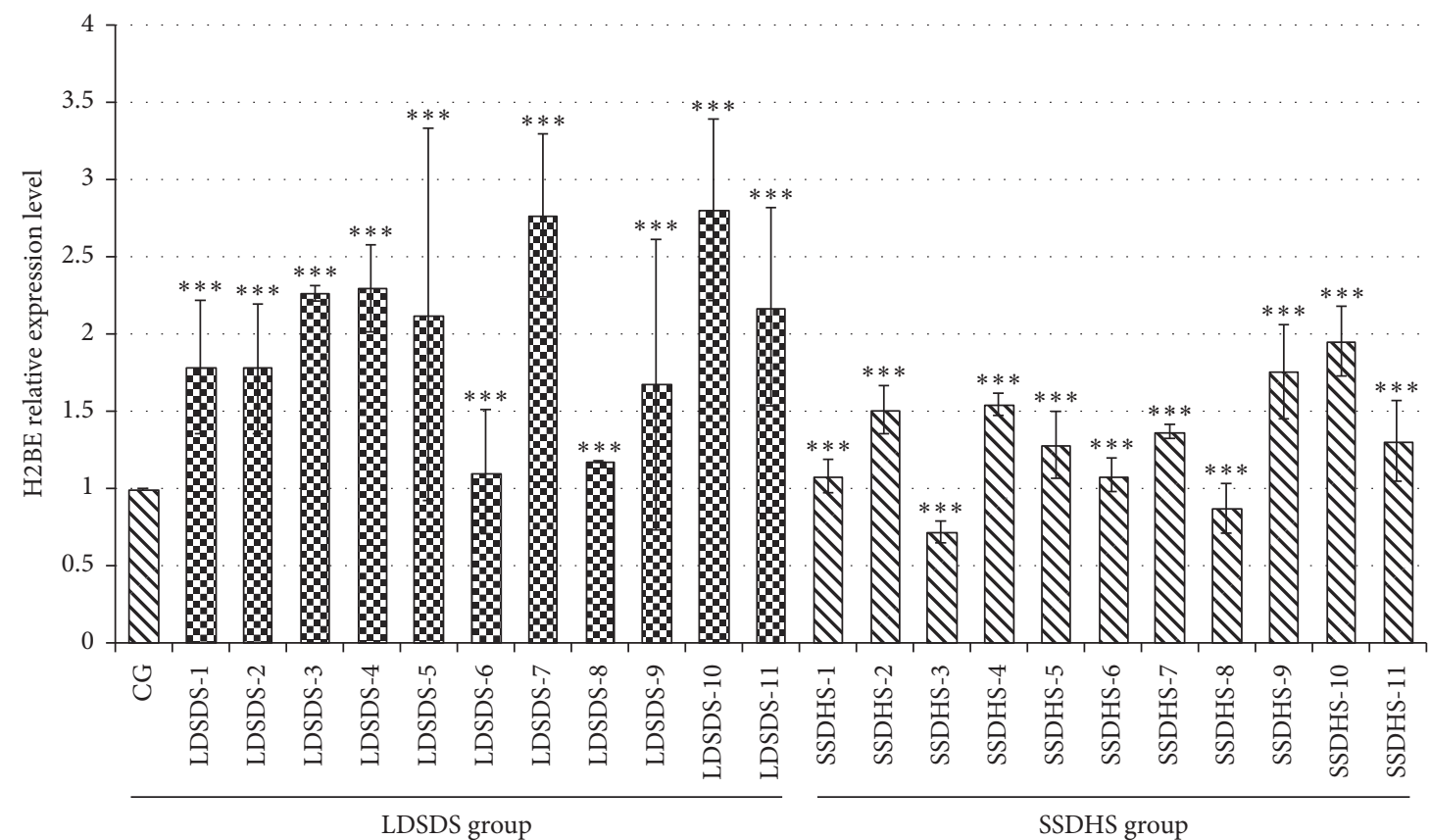

FIGURE 1: qRT-PCR validation of the H2BE gene in the three groups (LDSDS, SSDHS, and healthy group). The $X$-axis represents the samples studied in this study; the $Y$-axis represents the relative expression levels of genes. U6b gene was selected by our laboratory previously, which was used as a control. CG represents the healthy group. All data are from three technical repeats $(n=3) .{ }^{* * *}$ represents the significance level of the one-way ANOVA test, $P=0.001$. Error bars denote $|S| D$.

the expression regulation of $\mathrm{H} 2 \mathrm{BE}$ on the cytokines, the protein-protein interaction networks between $\mathrm{H} 2 \mathrm{BE}$ and the four cytokines were constructed. The results demonstrated that H2BE, IL-2, IL-4, IL-10, and some other related molecules were correlated mainly through physical interaction (67.64\%), coexpression (13.5\%), and signaling pathway $(4.35 \%)$ (Figure 2). Furthermore, after analyzing the major relationships in the network, we found that $\mathrm{H} 2 \mathrm{BE}$ was indirectly associated with IL-4 and IL-10, and H2BE regulated the expression level of cytokines mainly through GATA3 (Figure 3). That is, the GATA3 gene acts as a bridge between $\mathrm{H} 2 \mathrm{BE}$ and cytokines and also showed a coexpression pattern with $\mathrm{H} 2 \mathrm{BE}$, IL-4, and IL-10. These remind us that GATA3 may play an important role in the regulation of $\mathrm{H} 2 \mathrm{BE}$ on cytokines expression, but this needs to be further researched.

\section{Discussion}

Hepatitis B is a hidden killer. People infected with a virus for decades may show only mild symptoms, which could be easily ignored and may ultimately develop into liver cirrhosis or even liver cancer [24]. So far, the direct-acting antiviral agents (DAAs) of western medicine still could not eliminate the virus completely [25]. Therefore, pretreatment is of great value in the treatment of CHB and liver cirrhosis. TCM has been widely used in $\mathrm{CHB}$ pretreatment since its unique advantages, such as low toxicity side effect, good curative effect, etc. [26]. TCM syndrome plays an important role in TCM treatment, and the same disease with different TCM syndromes in western medicine could receive different treatments in TCM [11]. However, due to the fact that TCMsyndrome is overrelying on experience, this greatly limits its application worldwide. Thus, excavating available biomarkers for TCM-syndrome diagnosis and elucidating the underline mechanisms of TCM-syndrome could greatly help the application of TCM all over the world.

Previous studies found that the imbalances of Th1/Th2 cells and their cytokines are important causes of CHB [27]. However, Th1 and Th2 levels are strongly associated with the cytokine levels elaborated above [21]. For different CHB TCM-syndromes, as the TCM-syndrome changes, the immune responses may also be changed due to the ratios of 


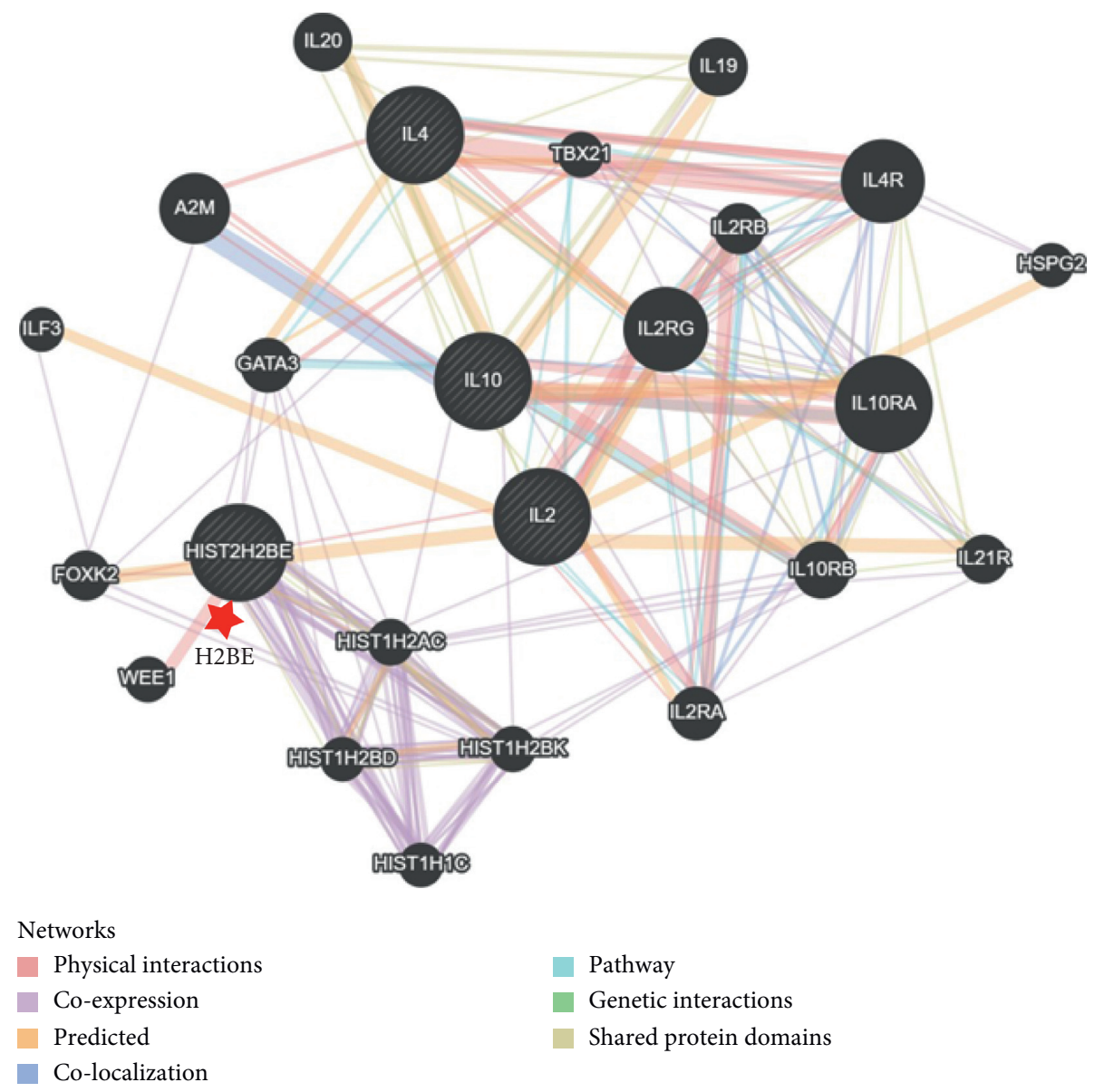

Figure 2: The protein-protein interaction networks between H2BE and the four cytokines. The networks between H2BE and the four cytokines were constructed by using the GeneMANIA database.

different cytokines are also changed. In this study, we found that the expression levels of almost all the examined cytokines were upregulated compared with the healthy group. Among the four cytokines, IL- 4 and INF- $\gamma$ showed statistical significance not only between the syndromes but also among the syndromes and healthy group, and these two cytokines may have potential as the biochemical indicators of the two TCM-syndromes. In addition, the results also demonstrated that almost all the cytokines were expressed much higher in LDSDS than in SSDHS, and this suggested that the immune response may be more intense in LDSDS than in SSDHS, and this result was also consistent with Lu et al. (2019) [11].

Histone is involved in many cellular processes such as transcriptional regulation, DNA replication, RNA splicing, etc., since the DNA is wrapped and interacts directly with histone proteins [28]. However, at present, a myriad of studies on histone mainly focus on histone modification, such as methylation, acetylation, phosphorylation, etc., rather than the effects generated by expression level changes $[18,29]$. In yeast cells, researchers found that overexpression histone H4 could suppress SIR2-induced inviability, while overexpression $\mathrm{H} 3$ did not [30]. Singh et al. [19] found that overexpression histone $\mathrm{H} 2 \mathrm{~A}$ resulted in a significant decrease in susceptibility towards drugs currently in use in Leishmania parasites, and thus pointing to its role in drug resistance. For H2BE, a study on breast cancer found that overexpression or downregulation of $\mathrm{H} 2 \mathrm{BE}$ could both lead to the decreased proliferation in breast cancer cell lines [31]. Furthermore, the expression level of H2BE was also associated with the cell function. That is, different cell types exhibit different expression levels of H2BE [32]. These indicated that $\mathrm{H} 2 \mathrm{BE}$ might play an important role in regulating the physiological function of cells. Interestingly, in this study, we found that the expression levels of H2BE in LDSDS were basically higher than that in SSDHS and showed an increasing tendency in $\mathrm{CHB}$ patients compared with the healthy group.

In the nucleosome, $\mathrm{H} 2 \mathrm{BE}$ is mostly exposed to the surface of the nucleosome compared with other nucleosome proteins [33, 34]. Hao [20] found that the subunit from 14th28th amino acid of $\mathrm{H} 2 \mathrm{~B}$ was the dominant epitope that could interact with Th cells and stimulate more cytokines secreted. In this study, we have also investigated the protein-protein interaction networks between $\mathrm{H} 2 \mathrm{BE}$ and the four cytokines, and the results displayed that GATA3 protein acts as a bridge between H2BE and cytokines and also showed a coexpression pattern with H2BE, IL-4, and IL-10. A myriad of studies reported that GATA3 could be expressed in large quantities in immune cells, such as T lymphocytes, NK cells, NKT cells, etc. [35-37]. Chen et al. [38] found that 


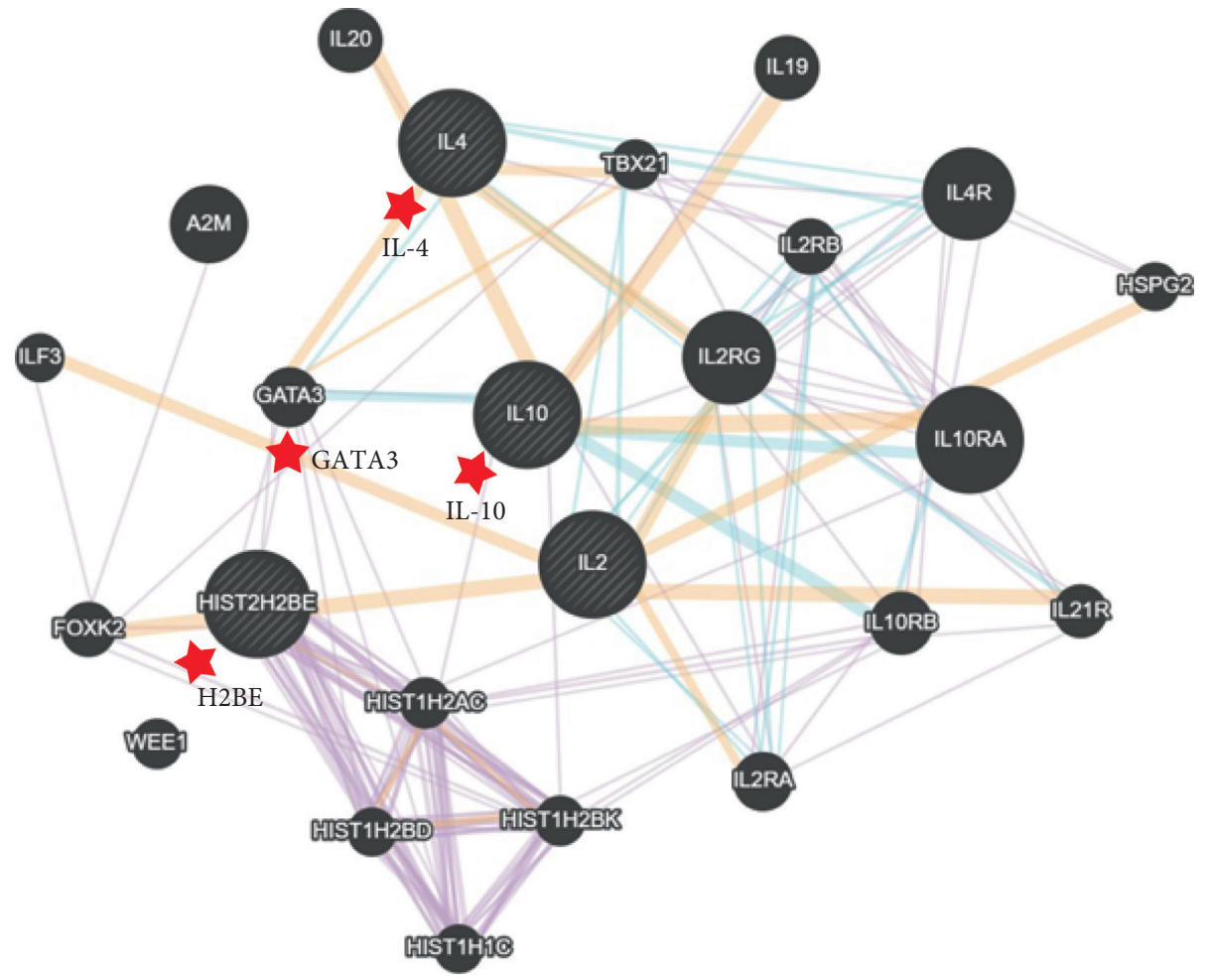

\footnotetext{
Networks

Physical interactions
Co-expression
Predicted
Co-localization
}

- Pathway

Genetic interactions

- Shared protein domains

FIGURE 3: The major relationships with $\mathrm{H} 2 \mathrm{BE}$ gene in the cytokines expression regulation. The networks between $\mathrm{H} 2 \mathrm{BE}$ and the four cytokines were constructed by using the GeneMANIA database.

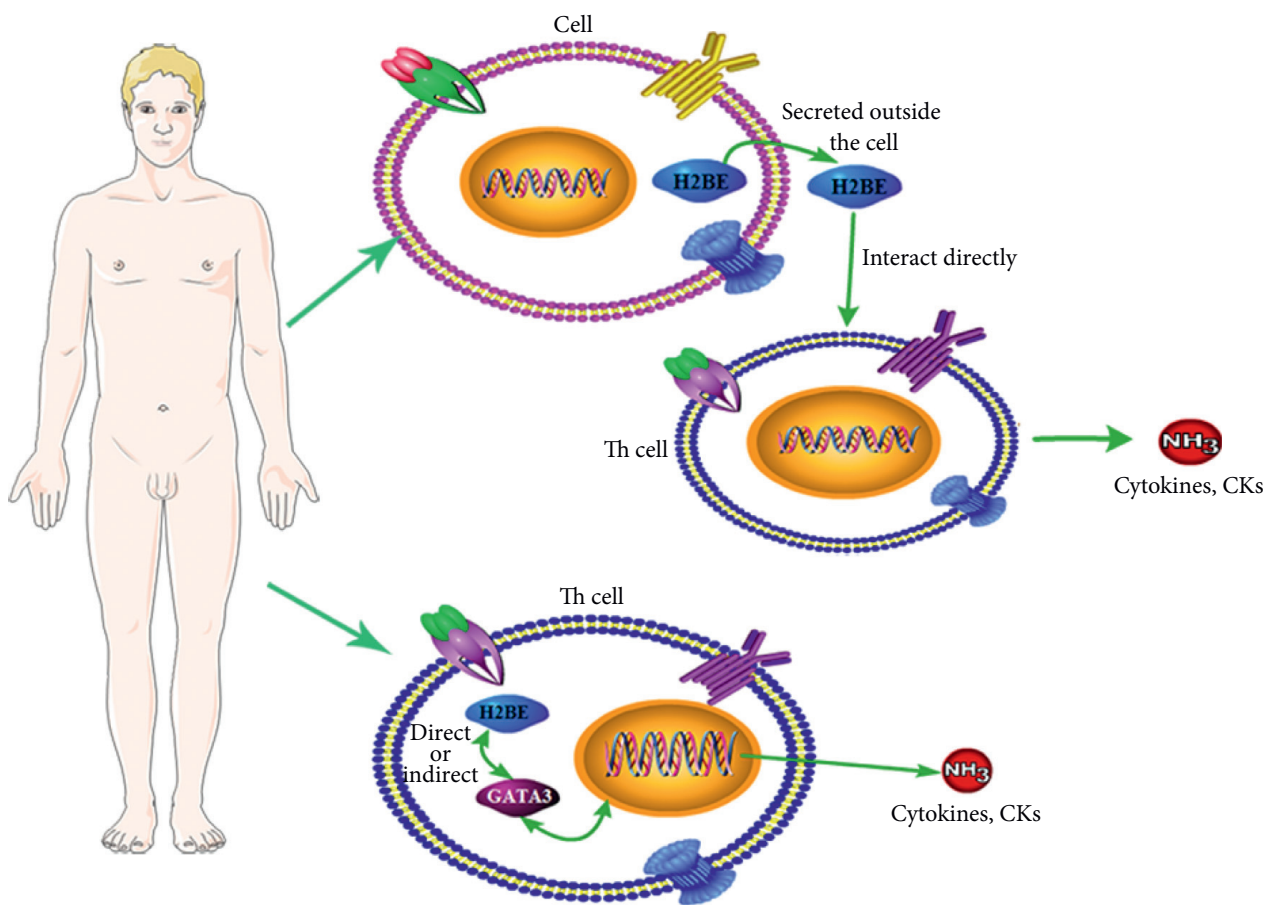

FIGURE 4: The possible regulatory pathways of H2BE involved in the secretions of cytokines. 
overexpression GATA3 could affect cytokine secretions of Th1 and Th2. Thus, we suggested that one other possible way for the expression regulation of Th related cytokines by $\mathrm{H} 2 \mathrm{BE}$ is through the direct or indirect interaction between H2BE and GATA3, among which GATA3 may be the intermediate node, with coexpression and other related pathways.

In summary, we believe that $\mathrm{H} 2 \mathrm{BE}$ regulates cytokine secretions via the following two ways, (1) H2BE could interact with Th cells directly through a dominant epitope way, and (2) the other way is by regulating the GATA3 gene directly or indirectly and then further regulating the cytokine secretions (Figure 4). However, further studies are needed to clarify the relative regulation mechanisms of $\mathrm{H} 2 \mathrm{BE}$.

\section{Data Availability}

The datasets used and/or analyzed during the present study are available from the corresponding author upon reasonable request.

\section{Ethical Approval}

The protocol has been approved by the Medical Ethics Committee of Affiliated Hospital of Chengdu University of Traditional Chinese Medicine, China. All study participants will sign two informed consent forms, one kept by the patients and the other kept by the researcher.

\section{Conflicts of Interest}

The authors declare no conflicts of interest.

\section{Authors' Contributions}

$\mathrm{CL}$ and $\mathrm{YZ}$ were the principal actors in this research, including sample collection and manuscript preparation. XL, $\mathrm{BL}, \mathrm{LW}$, and DW were mainly responsible for assisting in sample collection. QF and CJ provided fund support and scientific research guidance.

\section{Acknowledgments}

The authors are grateful to Shanhong Tang in the General Hospital of Chengdu Military Region for sample collection. This work was supported by the National Natural Science Foundation of China (81803976) and "Xinglin Scholar" Scientific Research Promotion Program for Academic Talents of Chengdu University of TCM (BSH2019018).

\section{References}

[1] Y. Jin, N. Geng, L. Zhao et al., "The prevalence of HBV infection: a retrospective study of 13-years in a public hospital of northeast China," Viral Immunology, vol. 33, no. 2, pp. 99-104, 2019.

[2] M. F. Yuen, D. S. Chen, G. M. Dusheiko et al., "Hepatitis B virus infection," Nature Reviews Disease Primers, vol. 4, p. 18035, 2018.
[3] L. S. Y. Tang, E. Covert, E. Wilson, and S. Kottilil, "Chronic hepatitis B infection," JAMA, vol. 319, no. 17, pp. 1802-1813, 2018.

[4] S. Lanini, A. Ustianowski, R. Pisapia, A. Zumla, and G. Ippolito, "Viral hepatitis: etiology, epidemiology, transmission, diagnostics, treatment, and prevention," Infectious Disease Clinics of North America, vol. 33, no. 4, pp. 1045-1062, 2019.

[5] WHO Guidelines Approved by the Guidelines Review Committee, Guidelines for the Prevention, Care and Treatment of Persons with Chronic Hepatitis B Infection, World Health Organization, Geneva, Switzerland, 2015.

[6] Y.-F. Liaw, "Natural history of chronic hepatitis B virus infection and long-term outcome under treatment," Liver International, vol. 29, pp. 100-107, 2009.

[7] P. Hao, F. Jiang, J. Cheng, L. Ma, Y. Zhang, and Y. Zhao, "Traditional Chinese medicine for cardiovascular disease," Journal of the American College of Cardiology, vol. 69, no. 24, pp. 2952-2966, 2017.

[8] Y.-H. Liao, C.-I. Li, C.-C. Lin, J.-G. Lin, J.-H. Chiang, and T.-C. Li, "Traditional Chinese medicine as adjunctive therapy improves the long-term survival of lung cancer patients," Journal of Cancer Research and Clinical Oncology, vol. 143, no. 12, pp. 2425-2435, 2017.

[9] J. Li, "Clinical research progress in integrated traditional Chinese and Western medicine therapy for HBV-related liver failure," Journal of Clinical Hepatology, vol. 31, no. 1, pp. 42-47, 2015.

[10] A. Lu, M. Jiang, C. Zhang, and K. Chan, "An integrative approach of linking Traditional Chinese Medicine pattern classification and biomedicine diagnosis," Journal of Ethnopharmacology, vol. 141, no. 2, pp. 549-556, 2012.

[11] Y. Lu, Z. Fang, T. Zeng et al., "Chronic hepatitis B: dynamic change in Traditional Chinese medicine syndrome by dynamic network biomarkers," Chinese Medicine, vol. 14, p. 52, 2019.

[12] H. Kang, Y. Zhao, C. Li et al., "Integrating clinical indexes into four-diagnostic information contributes to the Traditional Chinese Medicine (TCM) syndrome diagnosis of chronic hepatitis B," Scientific Reports, vol. 5, p. 9395, 2015.

[13] S. Sun, J. Dai, W. Wang et al., "Metabonomic evaluation of ZHENG differentiation and treatment by Fuzhenghuayu tablet in hepatitis-B-caused cirrhosis," Evidence-Based Complementary and Alternative Medicine, vol. 2012, Article ID 453503, 8 pages, 2012.

[14] A. Yukiharu, "The truth of the accident-some patients died from side efects of Xiao Chai Hu Tang," Journal of Tianjin University of Traditional Chinese Medicine, vol. 21, no. 1, pp. 47-48, 2002.

[15] M. Jiang, C. Lu, C. Zhang et al., "Syndrome differentiation in modern research of traditional Chinese medicine," Journal of Ethnopharmacology, vol. 140, no. 3, pp. 634-642, 2012.

[16] W. Zheng, Research on Correlations Among TCM Common Type of Syndromes and Laboratory Index in 1260 Patients with $\mathrm{CHB}$, Chengdu University of Traditional Chinese Medicine, Chengdu, China, 2011.

[17] C. J. Yang, H. W. Liu, and L. C. Wang, "Study on the differential gene expressions of chronic hepatitis B patients of gan depression pi deficiency syndrome and pi-wei damp-heat syndrome," Chinese Journal of Integrated Traditional and Western Medicine, vol. 32, pp. 1032-1037, 2012.

[18] P. B. Talbert and S. Henikoff, "Histone variants-ancient wrap artists of the epigenome," Nature Reviews Molecular Cell Biology, vol. 11, no. 4, pp. 264-275, 2010. 
[19] R. Singh, D. Kumar, R. C. Duncan, H. L. Nakhasi, and P. Salotra, "Overexpression of histone H2A modulates drug susceptibility in Leishmania parasites," International Journal of Antimicrobial Agents, vol. 36, no. 1, pp. 50-57, 2010.

[20] F. Hao, The Role of Nucleosome in the Pathogenesis of SLE and its H2B Peptide-Induced Immune Tolerance Therapies, Third Military Medical University, Chongqing, China, 2003.

[21] T. Chen, Y. Chen, W. Bao, and W. Lu, "T-lymphocyte subsets and Th1/Th2 cytokines in convalescent patients with EpsteinBarr virus-associated aplastic anemia," Hematology, vol. 25, no. 1, pp. 11-16, 2020.

[22] A. Jafarzadeh and F. Shokri, "TH1 and TH2 responses are influenced by HLA antigens in healthy neonates vaccinated with recombinant hepatitis B vaccine," Iranian Journal of Allergy, Asthma and Immunology, vol. 11, no. 4, pp. 308-315, 2012.

[23] Hepatobiliary Branch of Chinese Society of Traditional Chinese Medicine, "Standard of TCM syndrome differentiation for viral hepatitis (2017 version)," Chinese Journal of Integrated Traditional and Western Medicine on Liver Diseases, 2017.

[24] T. Tu, S. Bühler, and R. Bartenschlager, "Chronic viral hepatitis and its association with liver cancer," Biological Chemistry, vol. 398, no. 8, pp. 817-837, 2017.

[25] Q. J. Wu, W. L. Lv, J. M. Li et al., "Efficacy and safety of YinQiSanHuang antiviral decoction in chronic hepatitis B: study protocol for a randomized, placebo controlled, doubleblinded trial," Trials, vol. 21, no. 1, p. 482, 2020.

[26] Y. Y. Lu, Q. L. Chen, Y. Guan et al., "Study of ZHENG differentiation in hepatitis B-caused cirrhosis: a transcriptional profling analysis," BMC Complementary and Alternative Medicine, vol. 14, p. 371, 2014.

[27] M. Lee, M. A. Lee, S. K. Lee et al., "Expression of Th1 and Th2 type cytokines responding to HBsAg and HBxAg in chronic hepatitis B patients," Journal of Korean Medical Science, vol. 14, no. 2, pp. 175-181, 1999.

[28] C. Law, P. Cheung, and K. Adhvaryu, "Chemical "diversity" of chromatin through histone variants and histone modifications," Current Molecular Biology Reports, vol. 1, no. 1, pp. 39-59, 2015.

[29] T. C. Voss and G. L. Hager, "Dynamic regulation of transcriptional states by chromatin and transcription factors," Nature Reviews Genetics, vol. 15, no. 2, pp. 69-81, 2014.

[30] M. Matecic, S. Stuart, and S. G. Holmes, "SIR2-induced inviability is suppressed by histone $\mathrm{H} 4$ overexpression," Genetics, vol. 162, no. 2, pp. 973-976, 2002.

[31] S. R. Nayak, E. Harrington, D. Boone et al., "A role for histone $\mathrm{H} 2 \mathrm{~B}$ variants in endocrine-resistant breast cancer," Hormones and Cancer, vol. 6, no. 5-6, pp. 214-224, 2015.

[32] R. C. Molden, N. V. Bhanu, G. LeRoy et al., "Multi-faceted quantitative proteomics analysis of histone $\mathrm{H} 2 \mathrm{~B}$ isoforms and their modifications," Epigenetics \& Chromatin, vol. 8, p. 15, 2015.

[33] J. P. Portanova, J. C. Cheronis, J. K. Blodgett, and B. L. Kotzin, "Histone autoantigens in murine lupus. Definition of a major epitope within an accessible region of chromatin," Journal of Immunology (Baltimore, Md.: 1950), vol. 144, no. 12, pp. 4633-4640, 1990.

[34] C. Stemmer, J.-P. Briand, and S. Muller, "Mapping of linear histone regions exposed at the surface of the nucleosome in solution," Journal of Molecular Biology, vol. 273, no. 1, pp. 52-60, 1997.

[35] I.-C. Ho, T.-S. Tai, and S.-Y. Pai, "GATA3 and the T-cell lineage: essential functions before and after T-helper-2-cell differentiation," Nature Reviews Immunology, vol. 9, no. 2, pp. 125-135, 2009.

[36] T. Wang, A. L. Feldman, D. A. Wada et al., "GATA-3 expression identifies a high-risk subset of PTCL, NOS with distinct molecular and clinical features," Blood, vol. 123, no. 19, pp. 3007-3015, 2014.

[37] M. Anusree and B. Shashi, "Lower expression of GATA3 and T-bet correlates with down-regulated IL-10 in severe falciparum malaria," Clinical \& Translational Immunology, vol. 4, no. 11, p. 49, 2015.

[38] S. Chen, L. Wu, L. Peng, X. Wang, and N. Tang, "Hepatitis B virus $\mathrm{X}$ protein $(\mathrm{HBx})$ promotes ST2 expression by GATA2 in liver cells," Molecular Immunology, vol. 123, pp. 32-39, 2020. 